\title{
Assessment of Weight Stigma
}

\author{
Enrica N. Ruggs ${ }^{a} \quad E^{2}$ den B. King ${ }^{b} \quad$ Michelle Hebl $^{a} \quad$ Megan Fitzsimmons $^{a}$ \\ a Department of Psychology, Rice University, Houston, TX, \\ ${ }^{\mathrm{b}}$ Department of Psychology, George Mason University, Fairfax, VA, USA
}

\section{Key Words \\ Weight · Obesity · Stigma · Assessment · Methodology}

\section{Summary}

The purpose of this review article is to familiarize readers with the common methodologies used to assess weight stigma. This article explores the most frequent ways weight stigma is assessed, offers relevant empirical examples of each methodology, examines the strengths and weaknesses of each approach, and offers recommendations for strengthening research assessment of weight stigma for the future. Furthermore, this article highlights 4 dimensions that are important to consider when assessing weight stigma, regardless of the research methodology used.

\section{Assessment of Weight Stigma}

The dramatic increase in the number of heavy Americans presents an epidemic. Specifically, $74 \%$ of the US population currently can be categorized as overweight or obese [1], and this increase has been particularly rampant in the last decade [2]. As the prevalence increases, so too has the amount of research being conducted that focuses on weight issues, and much of this research (although not all) can be categorized as adopting a medical or social perspective. From a medical perspective, researchers are interested in causes and consequences of being heavy, as well as strategies for successful weight reduction. Clearly, a solid body of research has identified many of the medical concomitants of obesity [e.g., 3-4]. From a social perspective, which is the perspective adopted in the current review, researchers typically are interested in the pervasiveness and impact of weight stigmatization or the extent to which heavy individuals experience stereotypes, prejudice, and dis- crimination across social settings [5]. Researchers have used a variety of methods to assess weight stigma. In this article, we briefly describe these methods and then discuss in more detail 3 particular issues that are critical to consider when conducting research on weight stigma, regardless of methodological approach. In addition to highlighting these critical issues, we will comment on the past utility and future promise of current methodological approaches in understanding weight stigma.

\section{Brief Review of General Methods of Assessing Weight Stigma}

To study weight stigma, researchers have employed a number of different methods, the most common of which have included: survey methods, questionnaires with experimental manipulations, laboratory experiments, and field studies. Often times, researchers also use a combination of these methods to answer specific research questions and/or to show triangulation across methodology. We will briefly review each of these methods and provide examples of how they are currently used in assessing weight stigma. While these methods can be used to study obesity across the lifespan, the majority of research that we review in this paper focuses on adulthood obesity. Our goal is not to be comprehensive in reviewing every single study that has been conducted according to different methodologies; rather, we illustrate a few examples of the types of studies that have been conducted and findings that have been revealed in an attempt to give readers an idea about the distinctions between methodologies and their strengths and limitations.

\section{Survey Methods}

Surveys are generally self-report methods of collecting data, and are extremely useful in elucidating the stereotypes individuals hold concerning those who are heavy. Stereotypes involve the generalizations people make about the 'typical'

\section{KARGER}

Fax +497614520714

Information@Karger.de

www.karger.com
(C) 2010 S. Karger GmbH, Freiburg

Accessible online at:

www.karger.com/ofa 
characteristics of an individual because of his or her membership in a group. Thus, for instance, survey methods have revealed that people tend to view heavy individuals as also being unattractive, unprofessional, lazy, sloppy, undisciplined, and unhealthy [6-12]. One particular measure, The Fat Stereotypes Questionnaire (FSQ), an 18-item scale (9 duplicate items concerning thin people and fat people), has been used to demonstrate that negative stereotypes about obesity are present in both adults and children [13]. Among a sample of 178 parents, alpha coefficients for this scale ranged from 0.65 (mothers) to 0.69 (fathers). These reliability estimates fall below the conventional accepted reliability which is greater than 0.70; therefore, researchers should take caution before using this measure with adult samples.

Survey methods not only have been helpful in understanding stereotypes, they also have been critical to understanding the prejudices that people hold toward heavy individuals. Prejudice encompasses the attitudes that people have toward an individual because of his or her membership in a group, and indeed, many people hold strong prejudices toward those who are heavy. For instance, survey results have revealed that individuals would rather endure adverse life events such as being divorced, unable to have children, or giving up a year from their life than be heavy [11]. People also hold stronger prejudices toward those who are heavy to the extent that they believe being heavy is a controllable condition. In fact, one scale that has been particularly helpful in assessing prejudicial attitudes that people have toward those who are heavy is the Anti-Fat Attitudes (AFA) scale, a 13-item assessment which is comprised of 3 factors concerning the extent to which people i) dislike those who are heavy $(\alpha=0.84)$, ii) fear being heavy $(\alpha=0.79)$, and iii) belief that being heavy is a controllable condition $(\alpha=0.66)$ [14]. Despite the somewhat low reliability reported for the willpower (iii) sub-scale, this sub-scale was positively correlated with the dislike subscale $(\mathrm{r}=0.43$, $\mathrm{p}$ $<0.001$ ), whereas the fear of being heavy sub-scale was uncorrelated with both dislike and willpower sub-scales. These findings suggest that beliefs about controllability of weight and denigration of heavy people are directly in concert with one another. Further work by the author provides some evidence of construct validity for these 2 sub-scales. For instance, research showed that when participants believed excessive weight was the result of genetics and metabolism, scores on the willpower and dislike sub-scales were significantly lower than participants in the control group. Taken together, the evidence of reliability and validity suggest that the AFA is a psychometrically sound measure.

Crandall's AFA scale is probably the most popular survey method used to examine prejudicial attitudes toward heavy individuals. As such, its use is often seen in conjunction with other methods throughout the literature. However, other instruments have been developed to examine anti-fat attitudes. For instance, the Anti-Fat Attitudes Test (AFAT), a 47-item scale, was developed to capture a different aspect of attitudes related to weight [15]. This measurement consists of 3 subscales: i) social/character disparagement, ii) physical/romantic unattractiveness, and iii) weight control/blame. The developers of AFAT did not provided information concerning the reliability for these sub-scales or the overall scale. They did, however, show that people with no family history of obesity scored higher on the AFAT than people with family history of obesity, and people with no obese friends also scored higher on this scale than people with obese friends. These findings offer some support for the construct validity of this scale. The AFAT may be useful for researchers interested in capturing multiple aspects of individuals' attitudes and cognitions concerning weight. Both the AFA and the AFAT have a subscale that deals with weight controllability. These scales explore people's attitudes concerning the cause of obesity (e.g., lack of exercise). Another measure was developed to specifically examine people's attitudes concerning the possible causes of obesity. This scale, called The Causes of Obesity Scale, is a 31-item measure that contains 3 sub-scales: i) internal causes $(\alpha=0.84)$, ii) physical causes $(\alpha=0.63)$, and iii) social causes $(\alpha=0.60)$ [16]. The internal causes sub-scale of the Causes of Obesity Scale has decent reliability; however, the other 2 sub-scales do not $(\alpha<0.70)$. Therefore, the internal causes sub-scale may be a beneficial tool for researchers to use as a follow-up measure to either the AFA or AFAT to gain more information concerning people's anti-fat attitudes.-

Prior to the development of the AFA, the Attitudes Toward Obese Persons Scale (ATOP) and the Beliefs About Obese Persons Scale (BAOP) were developed [17]. In the original study, these measures were given to samples from 3 populations: members of the National Association to Advance Fat Acceptance (NAAFA), graduate students, and undergraduate students. The ATOP, a 20-item scale, had a reliability ranging from $\alpha=0.84$ to 0.80 between the populations, while the BAOP, a 10-item scale, had a reliability ranging from $\alpha=0.65$ to 0.82 . When used in later research, the BAOP has shown similar reliability [18]. The benefit of using these 2 scales simultaneously is that it allows researchers to capture information concerning both stereotypes (BAOP) and prejudices (ATOP). However, due to the low levels of reliability that have been reported for the BAOP, we recommend that researchers pre-test this measure and check for acceptable reliability above 0.70 prior to implementing it in a study.

While many researchers develop their own measure to meet the specific needs of a research question, others develop measures because they feel the previous measures are deficient or inadequate in some way. For instance, while the AFA is one of the most (if not the most) highly cited and replicable survey assessment used in the weight stigma literature, some researchers felt that one of the sub-scales (dislike of heavy people) is contaminated by questions that are awkwardly worded and prone to response bias [19]. In response to the AFA, the researchers developed their own 5-item anti-fat attitudes scale (AFAS). The AFAS showed a reliability ranging between $\alpha=$ 
0.72 and 0.80 across 4 studies. Additionally, the authors reported some support for construct validity. Despite the demonstration of psychometrically sound properties of the AFAS by the developers, this scale did not gain popularity by researchers, and is not often seen throughout the literature.

More recently, researchers have made attempts to develop psychometrically sound instruments that can aid researchers in examining prejudicial attitudes toward heavy individuals. For example the Universal Measure of Bias (UMB), a 20-item scale which is designed to capture underlying mechanisms associated with bias across different targets including obese targets, was recently developed [20]. This measure was composed using broadly phrased items in which bias category words such as 'fat' or 'gay' could be switched out with each other and the items still made sense. The authors tested 3 versions of this measure, including bias against heavy individuals (UMBFAT), bias against gay and lesbian individuals (UMB-GAY), and bias against Muslim individuals (UMB-MUS). When testing the psychometric properties of this new scale, researchers found that the UMB-FAT has adequate reliability ( $\alpha=0.87$ ). In addition, support for construct validity for the UMB-FAT scale was shown through the associations of this scale with both the AFA $(r=0.58)$ and ATOP $(r=0.50)$. This new measure offers potential for widespread use throughout the field.

Survey methods have highlighted that even those who interact often and closely with heavy individuals are susceptible to holding stereotypes and prejudicial attitudes toward them [e.g., 21]. In one survey, for instance, $50 \%$ of surveyed physicians characterized heavy patients as awkward, unattractive, ugly, and noncompliant [9]. In another survey, actual primary care physicians indicated that they would like their jobs less if the typical patient they saw was a heavy patient, that they would be less motivated to help and spend as much time with heavy than non-heavy patients, and that they did not think their heavy patients would be as likely to follow medical advice as would their thinner patients [22]. Such survey methodology is important in potentially showing that the quality of social interactions and medical attention, just to name a couple of examples, may be incredibly and negatively marred by the weight of an interactant.

Up to this point, we have discussed how survey methodology has been used to understand the perspective of those who stereotype or hold prejudices against heavy individuals. However, this research method also has revealed heavy targets' perceptions of attitudes regarding, and self-reported experiences due to their weight. In general, such research shows that heavy individuals consistently report being stigmatized, whether the perceivers and/or interactants are family members, doctors, co-workers, or classmates [for review see 23]. In fact, $54 \%$ of the large sample reported experiencing fairly severe weight stigma (i.e., derogatory jokes, being denied promotions or employment due to weight) from co-workers, and $45 \%$ reported experiencing weight stigma from employers. Survey research from the perspective of the target has also shown a surprising finding; namely, heavy individuals also stigmatize others on the basis of weight and do not benefit from ingroup identity, as do women, ethnic minorities, or many other ingroup members [14]. A new 11-item measure, the Weight Bias Internalization Scale (WBIS) $(\alpha=0.90)$ has shown that heavy individuals internalize the weight stigma that has been projected to them. While the WBIS was shown to be related to AFA, it does not appear to be an overlapping construct $(r=0.30)$ [24].

Another survey measure used to examine the target's perspective is the Perception of Teasing Scale (POTS) [25]. POTS is a revision of the Physical Appearance Related Teasing Scale (PARTS). The authors felt PARTS needed to be revised because it focused primarily on teasing related to physical appearance without taking into account perceptions of teasing that may be related to the target's abilities/competencies. POTS takes this into account, and consequently it is an 11-item scale that contains 2 factors: weight-related teasing (6 items) and competency teasing (5 items). After testing this scale on college-age females, researchers reported adequate reliability for both subscales ( $\alpha=0.88$ and $\alpha=0.84$ respectively).

The strengths of survey research to examine weight stigma include the fact that, in most cases, it is a relatively easy methodology to adopt. It is typically not difficult to find scales, collate materials, distribute surveys, and get responses. Given that survey methodologies often rely on convenience samples participating in either a paper survey or one distributed on the Internet, it is relatively easy to recruit individuals for what is typically a study that takes somewhere between 20-60 min. Furthermore, survey methodology allows access to both perceivers and targets' perspectives concerning weight stigma. Researchers who opt to use survey methodology should be sure to use measures that are psychometrically sound (i.e., measures with alpha coefficients greater than 0.70). Those who choose to use established measures with reported low reliability, adaptations of established measures, or develop their own measures should always be sure to pre-test these measures and calculate reliability to ensure that it is acceptable before proceeding on with the actual study.

The limitations of survey research, however, include the fact that this methodology relies primarily on self-reporting which is often biased. This is especially the case with a topic such as weight stigma in which individuals may feel social norms or pressures to respond in socially desirable ways. Although survey methods typically provide information on stereotypes and prejudices, this methodology does not do a very good job of capturing information regarding actual behaviors. Sometimes surveys will require people to report on their actual behaviors, and there have been some fascinating results that have emerged. For instance, parents of heavy college students are less likely to provide financial support for their children than parents of non-heavy students [26]. However, reports of behaviors are the exception and not the norm for survey research, and they do not necessarily provide reliable ac- 
counts of behavior. At best, information may be collected concerning behavioral intentions, and researchers may be able to postulate how one might behave during an interaction; however, these speculations may not always be accurate. Therefore, it is important for the assessment of obesity to extend beyond survey methodology in order to capture information concerning actual behaviors. To do this, researchers often use laboratory studies or field studies.

\section{Questionnaires with Experimental Manipulations}

Another common methodology used to assess weight stigma is the use of questionnaires (often either paper-and-pencil- or internet-based) that contain an experimental manipulation involving weight. The experimental manipulation may take on many different forms including (but not limited to): the use of pictures or photographs, vignettes, or category words. Participants are typically presented with only one of several different formats. An example of such a methodology is depicted in a study in which physicians reviewed a medical profile of a patient and evaluated it by expressing their attitudes about the patient as well as the treatments that they would prescribe [22]. The researchers manipulated how heavy the patient was depicted and found that the physicians reacted much more negatively toward the heavier patients than their thinner counterparts. In another example of this methodology, participants viewed targets who varied in size, and made ratings about them [7-8]. In one study specifically looking at the intersection between size and age, the researchers used an experimental manipulation in which different body sizes were morphed onto the same women's faces depicted at either 20, 40 , or 60 years of age. They found a prevalent and consistent pattern of obesity stereotyping across the lifespan. In another, very recent study, authentic managers were asked to assign new employees (depicted in a photo as either heavy or not heavy) to sales territories based on reading an information sheet with otherwise standard information [27]. Results revealed that managers were less likely to hire and more likely to assign poor sales territories to the obese individual, and these results were even more disparate if the obese individual was a woman versus a man.

The main strength of the questionnaire methodology is that it allows researchers to capitalize on the simplicity of the survey methodology. Not only is this method of assessment a relatively easy way to collect data without complication but the experimental manipulations additionally allow researchers to draw causal inferences about the results. If the only difference between conditions is the manipulated weight of a target, then researchers can deduce that weight is actually driving the observed results, unlike survey methodology in which inaccurate perceptions or inaccurate reporting may be responsible for any given set of results. While this method may have some benefits over the general survey, there are limitations of this type of assessment as well. In particular, responses on questionnaires that participants make regarding heavy individuals may be very different from the responses that they make when interacting face-to-face with heavy individuals. The potential lack of external validity does not arise necessarily from inaccurate perceptions or inaccurate reporting but rather from the lack of context that is created only when people actually interact with stigmatized targets (i.e., heavy individuals). Specifically, the dynamic interplay, interdependence, and emotions between perceiver and target are missing [for review see 28]. To better understand these dynamics, we generally need more interactional methods. We now move to considering more interactional types of methodologies used to assess weight stigma.

\section{Laboratory Experiments}

The use of laboratory experiments offers researchers another common method used to assess weight stigma. In a typical laboratory experiment, participants are shown the same stimulus material with the exception of a weight manipulation. That is, participants might enter a laboratory and believe that they are taking part in a study on job interviews. In the course of the study, they are shown a video of a job applicant [29], or might actually interact with a candidate [30] who is either heavy or not. Such studies triangulate with survey research in showing the negative stereotypes and prejudice that participants hold toward heavy individuals but they also begin to document discrimination which is the negative behavioral treatment that is displayed toward an individual because of his or her membership in a group. One recent laboratory experiment that effectively demonstrates weight discrimination examined the evaluations that job trainers made of job trainees [31]. Specifically, participants were randomly assigned to play the role of either a trainer or trainee, and then the trainer was shown a manipulated photograph of either a heavy or average weight trainee and told that this was the individual he or she would be training through the use of computer-mediated training. Results showed that trainers evaluated the training more negatively (i.e., they did not feel the training was successful at preparing the trainee for the task) when the trainee was heavy versus not heavy. Furthermore, trainees actually performed more poorly when the trainers believed them to be heavy versus not heavy. Such research effectively shows that people have negative expectations and these translate into negative evaluations of and behaviors directed toward heavy individuals. These types of findings also suggest that the treatment provided to heavy individuals (in this case, individuals whom were believed to be heavy) can negatively impact the target individual. In assessing weight stigma, laboratory experiments use manipulations other than just those involving weight. For instance, researchers have examined how others' attitudes about obesity influence participants [32]. They manipulated the favorability of attitudes that others had toward obese people and found that perceived consensus about positive attitudes toward obese others significantly influenced participants to also demonstrate more positive attitudes. 
Although many laboratory studies manipulate weight to examine the stigmatizer's perspective, laboratory experiments can also be used to examine the target's perspective. For instance, researchers have manipulated feedback such that heavy (and not heavy) female participants received either positive or negative social feedback from a male evaluator [33]. Heavy (but not the thinner) women who received this negative feedback attributed the feedback to their weight (i.e., their own fault) rather than to the prejudices of their evaluators. Unlike most stigmatized targets (i.e., women, ethnic minorities) who are able to attribute negative feedback to others, heavy targets believed that the negative feedback was justified, and they experienced negative mood and lower selfesteem. In another laboratory study examining the target's perspective, interaction partners rated heavy women's social skills when they were actually visible versus not visible, and believed themselves to be either visible versus not visible [34]. The results showed a main effect for perceived visibility such that targets compensated by acting more likable when they believed that they were visible to their interactants, whether or not they actually were visible. Such results suggest that heavy targets are aware of the obesity stigma and actively engage in compensatory behaviors to try to mitigate the discrimination to which they are typically subjected.

The main strength of laboratory experiments is that they often have high levels of internal validity; that is, they allow researchers to make strong inferences concerning causality when the manipulations are effectively applied, procedures are standardized, and extraneous variables are controlled. Moreover, laboratory studies allow researchers to directly measure behaviors as opposed to relying on perceptions of behaviors; therefore, researchers can have some confidence in believing that certain behaviors actually occurred (or will occur) and that they result directly from the weight stigma. The studies using laboratory experiments that are currently seen in the research literature have provided us with useful information concerning both actors' reactions to weight manipulations. They have also allowed for the examination and recording of targets' behavior when interacting with actors. When combined with survey methodology, laboratory experiments allow researchers to examine possible underlying mechanisms for why weight stigmatization occurs (e.g., are individuals with high AFA scores those likely to respond most negatively to heavy targets?), as well as explore the thoughts and attitudes of targets in the midst of discrimination. While laboratory experiments also allow inferences concerning causality, these designs are not without limitations. The first limitation is that such studies are often much more complex studies to conduct than those previously described. Laboratory studies are typically run one participant at a time, and sometimes involve the necessity for research confederates and deception. A second limitation of this methodology concerns realism. Many experimental designs involve participants going to a laboratory to participate in a psychology study, which may create suspicion about the nature of the study. It also may cause some participants to respond or behave differently than they would if they were completing an anonymous survey or engaging in an interaction in a realistic setting.

\section{Field Studies}

The final methodology that we consider involves field studies which are studies on weight stigma that typically are i) conducted outside of the laboratory confines, ii) involve social interactions, and iii) often use of some experimental manipulation. In a standard field study, participants interact with a heavy (or non-heavy) target, during which time both verbal and nonverbal behaviors are recorded. For instance, researchers recently examined the customer service that obese individuals received. For the first 2 studies, confederate customers either wore (or did not wear) an obesity prosthesis and entered retail stores looking for customer service [35]. The results in both of these studies revealed that all customers were greeted and offered assistance, regardless of their weight. However, personnel interacted with heavy (versus non-heavy) customers by being less friendly, terminating interactions sooner, smiling less, and speaking fewer words. In a third related study, the researchers showed the organizational implications of such negative personnel treatment toward heavy individuals by examining the experiences of authentically heavy and non-heavy individuals. Shoppers who were exiting retail stores were asked about their experiences and categorized by experimenters as being either heavy or not. Heavy shoppers who experienced negative interpersonal behaviors (versus those who did not) spent less money than they had intended initially to spend, did not plan to return soon for a future visit, and indicated they would not strongly recommend the store to others.

There are clearly fewer studies assessing the weight stigma being conducted with field study methodology than other methodologies. However, the strength of using field studies as an assessment tool in understanding weight stigma is that this methodology captures behaviors that unfold when stigmatizers and targets interact interdependently in actual social interactions. Similarly, this methodology, as compared to explicit survey and laboratory studies, may allow researchers to examine more candid interactions between stigmatizers and targets because interactants are less likely to be able to 'control' their responses if they do not even know that they are being examined. Like laboratory studies, field study methodology also allows researchers to infer causality from results. This method provides a host of rich information and offers the possibility of high levels of external validity; however, it is probably the least often used of the 4 methods we describe. There are several reasons for this, one of which is that field studies are typically much more complex and require more manpower and hours to conduct than do other methodological alternatives. Another limitation concerns the ethical issues that may arise when researchers manipulate real-world situations in which a 
stigmatized and non-stigmatized individual interact. If the interaction is going to be authentic, then it is sometimes necessary that individuals (at least either stigmatizer or target) remain naïve as to the fact that they are in a study and this raises ethical issues concerning informed consent. Although the ethical concerns should certainly be considered, a properly designed field study can provide an abundance of useful and novel information concerning the nature of weight bias that authentically occurs in social interactions. Therefore, we are hopeful and encourage future researchers who study weight bias to consider conducting more field studies and other interactive methods [see also 28].

\section{Additional Approaches}

Although we have defined and addressed 4 of the most common methods used to assess weight stigma, it is important to note that these approaches are not exhaustive. For instance, the use of implicit measures is gaining in popularity. An excellent review of the use of implicit measures for assessing weight stigma was recently published [36]; therefore, we will only provide a very brief overview here. Implicit methods are used to assess the attitudes people privately hold but may not explicitly express due to social norms. Hence, implicit measures reflect attitudes that people may be unwilling to express or unaware that they possess. One common measure used in assessing implicit attitudes regarding weight stigma is the Implicit Associations Test (IAT). For instance, the IAT has been used to show that health professionals who focus on obesity research are not immune to having biases against the individuals whom their research helps. Specifically, one study showed the health professionals have an implicit anti-fat bias, and they also endorse negative stereotypes about heavy individuals [37]. Others have shown that relatively few differences emerge between individuals implicit and explicit associations toward weight [38]. For many other stigmas such as race, implicit and explicit attitudes differ such that negative explicit attitudes are not expressed [e.g. 39]. This finding that implicit and explicit attitudes are similar for the weight stigma speaks to the severity of this particular stigma. It suggests that people believe it is okay to openly stigmatize individuals due to weight. While the IAT is a common measure of implicit bias, not all studies show small differences between implicit and explicit associations. As with race, some weight IAT studies have shown a non-significant relationship between implicit and explicit attitudes [36]. This lack of relation between the IAT and known measures of anti-fat attitudes lead some to question the criterion validity of this measure. That is, does the IAT actually measure what it is intended to measure (i.e. attitudes toward weight)? Proponents of the IAT argue that these findings are due to social norms, and that this is the benefit of the IAT, to show prejudices that people are not willing to explicitly express. While there is no clear solution to this problem, researchers who use the IAT should be aware of its validity issues.
Also, recent weight stigma research has begun to look at the functional anatomic correlates of weight stigma using the novel methodological tool of functional magnetic resonance imaging (fMRI) [40]. For instance, one group of researchers recently found that the prefrontal regions of the brain, which are thought to be associated with inhibition and control, were non-responsive to overweight stimuli [40]. These findings support previous weight stigma research by suggesting that people do not necessarily inhibit their negative attitudes toward heavy individuals because they believe that weight is something that is controllable. Researchers have also gained valuable information concerning weight stigmatization through various content analyses of media representations of the stigma. These analyses have shown the prevalence of the portrayal of overweight or obese characters as the target of ridicule and humor, and rarely the target of romance or affection [21]. These analyses are also important because they highlight the portrayal of weight as a controllable issue in advertisements and news coverage [21]. fMRI studies and content analyses are just two additional approaches that can be employed.

Table 1 highlights the key factors that have been discussed for each method reviewed. It is important to note that methodologies are not necessarily independent from or orthogonal to each other. Laboratory studies sometimes include surveys, and some studies using questionnaires with experimental manipulations are conducted in the field. As mentioned earlier, combining various methods may allow for an examination of not only the occurrence and prevalence of stigmatization, but also the motivations and mechanisms driving this process. Clearly, there are other sorts of variations on the methodologies that we presented, and we did not attempt to be exhaustive; rather, we intended to give the readers a general understanding and some examples of the most common types of methodologies that are used to assess weight stigma.

\section{Important Dimensions to Consider when Assessing Weight Stigma}

Now that we have described the typical methodologies used in assessing weight stigma, we turn to a discussion of some of the critical issues to consider in conducting such research, regardless of the adopted methodological approach. That is, one can review the assessment of weight stigma by considering each of the different methodologies, as we have done thus far, or one can consider the critical methodological questions that should be considered regardless if one is conducting survey, questionnaire, laboratory, or field research. We discuss what we consider to be 3 of such critical issues to consider, and describe how research has fallen short in fully considering these issues and how future research might compensate for past weaknesses. 
Table 1. Primary methodologies: uses, strengths, and weaknesses

\begin{tabular}{|c|c|c|c|c|}
\hline Method & Primary assessment & Strength & Weakness & Examples \\
\hline Surveys & $\begin{array}{l}\text { stereotypes, beliefs, } \\
\text { and prejudices }\end{array}$ & $\begin{array}{l}\text { relative ease of use } \\
\text { (development, data } \\
\text { collection, recruitment } \\
\text { of participants) }\end{array}$ & $\begin{array}{l}\text { relies primarily on self- } \\
\text { report which is often } \\
\text { biased; causality cannot } \\
\text { be inferred }\end{array}$ & $\begin{array}{l}\text { AFA (Crandall } 1994 \text { [14]) } \\
\text { AFAT (Lewis et al. } 1997 \text { [15]) } \\
\text { ATOP/BAOP (Allison et al. } \\
\text { 1991 [17]) } \\
\text { UMB (Latner et al. } 2008 \text { [20]) }\end{array}$ \\
\hline $\begin{array}{l}\text { Laboratory } \\
\text { experiments }\end{array}$ & $\begin{array}{l}\text { attitudes, behavior } \\
\text { and discrimination }\end{array}$ & $\begin{array}{l}\text { high levels of internal } \\
\text { validity; allow for direct } \\
\text { measure of behavior; } \\
\text { allows researchers to infer } \\
\text { causality }\end{array}$ & $\begin{array}{l}\text { complex to conduct; } \\
\text { more time consuming } \\
\text { than surveys or } \\
\text { questionnaires; may } \\
\text { suffer from lack of } \\
\text { realism }\end{array}$ & $\begin{array}{l}\text { use of manipulation techniques } \\
\text { such as videos, photographs, } \\
\text { prosthetics, etc. }\end{array}$ \\
\hline Field studies & $\begin{array}{l}\text { behaviors and } \\
\text { discrimination }\end{array}$ & $\begin{array}{l}\text { capture actual behaviors } \\
\text { as they occur in a real } \\
\text { environment; allows for } \\
\text { examination of authentic } \\
\text { interactions between } \\
\text { targets and perceivers; } \\
\text { allows researchers to infer } \\
\text { causality }\end{array}$ & $\begin{array}{l}\text { very complex to conduct; } \\
\text { ethical issues may arise } \\
\text { using this method }\end{array}$ & $\begin{array}{l}\text { observe actual interactions } \\
\text { between actors and } \\
\text { authentically heavy individuals } \\
\text { or individuals wearing obesity } \\
\text { prosthetics }\end{array}$ \\
\hline
\end{tabular}

\section{Issues Pertaining to Weight-Related Terminology and Manipulations}

There are many issues concerning terminology that we believe researchers must consider when conducting research on weight stigma. First, past research has revealed a great deal of disparities in the terminology that researchers use when assessing weight stigma. For instance, 'obese' is often used interchangeably with, or with very little distinction from, 'overweight'. To avoid using these terms incorrectly, in fact, we attempted to restrain from using either and simply choose the word 'heavy' instead. Second and similarly, 'overweight' suggests that people are actually over a weight that they should be but the standards by which such weights were decided are derived from the somewhat archaic Metropolitan Life Insurance tables produced in the 1940s, which was simply intended to indicate the size of people with the lowest mortality rates. Such 'ideal weights' [41] have not been replaced wholly because it is not clear what alternatives might be better [42]. One example of our reliance on these numbers shows researchers classifying individuals into an overweight category if they are as little as 5-10 pounds over the guidelines suggested by these life insurance tables [i.e., 33]. Then, these 'overweight' individuals are compared with individuals who are only 5 pounds fewer. It is astounding that results emerge but such research begs the question of what other cut-offs might be more appropriate or theoretically interesting to explore.
Some researchers rely on the body mass index (BMI) to define the constructs of overweight and obese, but this can also be problematic because it is only a surrogate measure and not a direct measurement of the excess accumulation of body fat that an individual has [42]. Yet it is important to note that the BMI may be the best measure we have at present. Third, another way in which the terminology can be differentially interpreted is whether heavy individuals are referred to with positive descriptors such as 'full figured' or more negative descriptors such as 'obese' or 'fat' [43]. While at extremes, researchers may be very good at identifying and defining what is thin versus heavy, the lines quickly become fuzzy when researchers are not addressing the extremes. Fourth, researchers should try to look at weight as a continuous variable rather than a dichotomy of overweight or obese versus average. It is possible that researchers may have missed a great deal of information (i.e., curvilinear relationships, whether the effect is driven by thinness or by being heavy) by only looking at 2 or sometimes 3 (i.e., thin, medium, large) different weight categories. Similarly, it is not clear if 'overweight' is viewed the same as 'obesity' because little research, particularly experimental, has articulated both of these categories. Fifth, manipulations of the weight stigma are sometimes done using pictoral representations (i.e., stick drawings, pictures of people varying in size) but this also makes it difficult to know exactly what size is being manipulated. Similarly, heavy people do not 
uniformly gain weight everywhere - some are pear shaped, some are apples, some are top heavy, and some carry excess weight in their thighs - but the pictoral representations are often limited in showing such variations so it is not clear at all from previous research if some types of heavy are more acceptable than others.

In sum, most research on weight bias could benefit from the use of more precise language and improved definitions and terminology to discuss variations in weight. We propose that directing future research toward this methodological issue would be very helpful to the study of weight stigma and would progress this area of research immensely.

\section{The Stigmatizer and/or Target Perspective}

The stigma of weight has been explored at least somewhat from both the stigmatizer and the target's perspective; however, research examining the stigmatizer's perspective is much more pervasive within the literature for a number of reasons, perhaps centrally because it is easier to conduct. That is, researchers can simply ask participants to give their opinions of and attitudes about heavy targets, show them pictures of heavy targets, and/or have them believe they will interact or actually interact with an individual who is either authentically heavy or heavy with the use of a prostheses. In these conditions, no or very few actually heavy individuals are needed to conduct the research. It is not surprising, then, that research on the stigmatizer's perspective has dominated the literature, revealing the why, when, and consistency across situations in which stigmatizers feel negatively toward heavy targets [e.g. $12,22,37,44]$. In fact, two recent meta-analyses have documented that employers and managers stigmatize heavy employees and subordinates across all stages of the employment cycle $[10,45]$.

Although the majority of research has adopted the stigmatizer's perspective, some research has adopted the perspective of stigmatized targets, most of which has involved survey methodology. Thus, research has revealed a great deal about the disparity in heavy versus non-heavy individuals' salary earnings, assigned sales territory, friendships and relationship networks, and body image and other psychological issues; and this research consistently reveals that when there are differences, heavy individuals experience more negative outcomes than do their non-heavy counterparts [for review see 21]. An obvious reason for the reliance on some methodologies more than others is that weight as a target variable cannot be manipulated - thus, researchers must be careful when drawing inferential differences between authentically heavy and nonheavy individuals. The results may have to do with the weight but other variables might also influence the results so researchers must exercise caution in their interpretations of such data.

In sum, there are scores of research questions that weight researchers have not been able to address because they have focused (and it is easier to focus) on the perspective of stigma- tizers. We propose future research should be conducted on each perspective using each of the different methodological approaches. Furthermore, what is almost missing entirely from the weight stigma literature is research that considers both perspectives simultaneously. An exception to this is a study that we described previously, which assesses store personnel's verbal and nonverbal behaviors toward non-heavy or heavy customers wearing obesity prostheses [35]. In addition to this perspective, the researchers also asked the customers themselves as well as observers of the interaction to indicate the extent to which they believed the interaction was tainted. The results showed consistency across perspective - customers and observers were both able to identify the discrimination that employers displayed toward them. This research is important both for what it does and does not show. The congruency in findings across perspective reveals that not only do stigmatizers discriminate but that this discrimination is aptly and accurately perceived by recipients and third-party observers. However, perceptual differences in perspective would also be interesting and might reveal important future directions for research [e.g. 34]. In fact, research on other stigmas has shown differences in perspectives [see 46], and such differences lay the groundwork for figuring out how to reconcile misperceptions and social interactional difficulties.

\section{Assessment of Attitudes and/or Behaviors}

Not only can researchers gain insights from the congruencies and incongruencies that emerge between stigmatizer and target perspectives but so, too, can such insights be garnered from the similarities and dissimilarities that emerge between the attitudes and behaviors that interactants express toward each other. Research on various stigmas has revealed disconnects between the explicit (often overt or verbal) and the implicit (often more subtle and nonverbal) behaviors that perceivers and targets express toward each other [for review see 47] but it is not clear whether such disconnects also exist for the weight stigma. On the one hand, the few studies that have assessed both attitudes and behaviors leads one to believe the disconnect also might exist with the weight stigma. Namely, research examining formal discrimination (a more overt, explicit type that often involves verbal statements) and interpersonal discrimination (a more subtle, covert type that often involves nonverbal behaviors) revealed that store personnel did not express overt types of discrimination or explicitly state negative attitudes toward heavy individuals but they did engage in more subtle, interpersonal forms involving nonverbal behaviors [35]. This pattern may reflect the possibility that individuals were able to manage successfully their overt but not subtle expressions. On the other hand, however, there is also research that suggests that the weight stigma acts differently from other stigmas and that it has some very pernicious qualities. For instance, those who are heavy get few ingroup benefits from being heavy and also stigmatize themselves and others for being heavy [14]. This type of ingroup denigration is not typi- 
cally seen for other stigmas (e.g. race, gender). In addition, unlike other stigmatized groups, few laws protect people on the basis of weight. This lack of protection potentially allows for greater congruency between stigmatizing attitudes and behaviors that may not be seen for other stigmatized groups.

In a review on social stigma, researchers have noted a relative abundance of survey and attitudinal studies and a dearth of interactive studies that focus on behaviors [28]. This imbalance seems to be particularly apparent in the weight stigma literature. We are hopeful that more interactive studies will be conducted on the weight stigma, and that they will include measures of both attitudes and behaviors. Collecting both sets of data allows researchers to examine potential incongruencies between attitudes and behaviors, as well as explicit and implicit behaviors, which ultimately may help researchers determine what types of weight stigma remediation strategies should be developed and implemented.

\section{Conclusions}

In this review, we have explored the 4 most common ways in which the obesity stigma is assessed. For each of these methodologies - surveys, questionnaires with experimental manipulations, laboratory experiments, and field studies - we have offered examples of the types of research findings that have been unveiled. In addition, we have considered both the strengths and weaknesses of each approach, and hope that future research will continue to use all of the approaches and triangulate findings across them to gain a more comprehensive view of how weight stigmatization occurs and how it may occur differently across diverse domains. In addition, we have outlined a few important methodological concerns we feel are critical for future weight stigma researchers to consider. In particular, we hope that future researchers will more carefully reflect on their choices of weight-related terminology and manipulations, select measures that are psychometrically sound, assess both the stigmatizer and target's perspective, and consider attitudes and behaviors simultaneously. Furthermore, we hope researchers will strive to incorporate more interactive methods in future weight stigma research [see 28]. The combination of interactive with non-interactive methods may allow researchers to make comparisons and gain significant insights concerning the same individuals' attitudes and behaviors.

Finally, we cannot conclude this article without also promoting future research that focuses on intervention programs. This article primarily highlights research showing that weight stigmatization does occur; however, research assessing obesity stigmatization needs to extend beyond simply showing that this stigma occurs in various domains and take the next leap toward examining strategies for reducing weight bias from the perspective of both targets and stigmatizers. Researchers have begun to take steps in examining remediation against weight stigma, and some strategies appear to be effective. For instance, influencing people's perceptions of social consensus toward heavy individuals has been shown to help increase positive attitudes to them [32]. Researchers should continue along this line of research and develop sound measures to test the effectiveness of remediation strategies. In short, it is crucial that sound research, regardless of methodology, continues to be conducted and that we strive toward a greater understanding and resolution of the stigma of obesity.

\section{Disclosure}

The authors declared no conflicts of interest.

\section{References}

1 World Health Organization: Obesity and Overweight, 2006. www.who.int/mediacentre/factsheets/ fs311/en/index.html.

- Finkelstein EA, Ruhm CJ, Kosa KM: Economic causes and consequences of obesity. Annu Rev Public Health 2005;26:239-257.

$\checkmark 3$ Bray GA: Medical consequences of obesity. J Clin Endocrinol Metable 2004;89:2583-2589.

4 Must A, Strauss RS: Risks and consequences of childhood and adolescent obesity. Int J Obes 1999; 23:S2-S11.

5 Dovidio JF, Major B, Crocker J: Stigma: introduction and overview; in Heatherton TF, Kleck RE, Hebl MR, Hull JG (eds): The Social Psychology of Stigma. New York, Guilford Press, 2000, pp. 1-29.

6 Cramer P, Steinwert T: Thin is good, fat is bad: how early does it begin? J Appl Dev Psychol 1998;19: 429-451.

7 Hebl M, Heatherton TF: The stigma of obesity in women: the difference is Black and White. Pers Soc Psychol Bull 1998;24:417-426.
8 Hebl MR, Ruggs EN, Singletary SL, Beal D: Perceptions of obesity across the lifespan. Obesity (Silver Spring) 2008;16:S46-S52.

9 Foster GD, Wadden TA, Makris AP, Davidson D, Sanderson RS, Allison DB, Kessler A: Primary care physicians' attitudes about obesity and its treatment. Obes Res 2003;11:1168-1177.

10 Roehling MV: Weight-based discrimination in employment: psychological and legal aspects. Pers Psychol 1999;52:969-1016.

11 Schwartz MB, Vartanian LR, Nosek BA, Brownell KD: The influence of one's own body weight on implicit and explicit anti-fat bias. Obesity (Silver Spring) 2006;14:440-447.

12 Teachman BA, Brownell KD: Implicit anti-fat bias among health professionals: is anyone immune? Int J Obes 2001;25:1525-1531.

13 Davison KK, Birch LL: Predictors of fat stereotypes among 9-year-old girls and their parents. Obes Res 2004;12:86-94.
14 Crandall CS: Prejudice against fat people: ideology and self-interest. J Pers Soc Psychol 1994;66:882-894.

15 Lewis RJ, Cash TF, Jacobi L, Bubb-Lewis C: Prejudice toward fat people: the development and validation of the antifat attitudes test. Obes Res 1997;5: 297-307.

16 Klaczynski PA, Goold KW, Mudry JJ: Culture, obesity stereotypes, self-esteem, and the 'thin ideal': a social identity perspective. J Youth Adol 2004;33: 307-317.

17 Allison DB, Basile VC, Yuker HE: The measurement of attitudes toward and beliefs about obese persons. Int J Eat Disord 1991;10:599-607.

18 Neumark-Sztainer D, Story M, Harris T: Beliefs and attitudes about obesity among teachers and school health care providers working with adolescents. J Nutr Educ 1999;31:3-9.

19 Morrison TG, O'Connor WE: Psychometric properties of a scale measuring negative attitudes toward overweight individuals. J Soc Psychol 1999; 139:436-445. 
20 Latner JD, O'Brien KS, Durso LE, Brinkman LA, MacDonald T: Weighing obesity stigma: the relative strength of different forms of bias. Int J Obes 2008;32:1145-1152.

21 Puhl RM, Heuer CA: The stigma of obesity: a review and update. Obesity (Silver Spring) 2009;17: 941-964.

22 Hebl MR, Xu J: Weighing the care: physicians' reactions to the size of a patient. Int J Obes 2001;25 1246-1252.

23 Puhl RM, Brownell KD: Confronting and coping with weight stigma: an investigation of overweight and obese adults. Obesity (Silver Spring) 2006;14: 1802-1815.

24 Durso LE, Latner JD: Understanding self-directed stigma: development of the Weight Bias Internalization scale. Obesity (Silver Spring) 2008;16:S80-S86.

25 Thompson JK, Cattarin J, Fowler B, Fisher E: The perception of teasing scale (POTS): a revision and extension of the physical appearance related teasing scale (PARTS). J Pers Assess 1995;65:146-157.

26 Crandall CS: Do heavy-weight students have more difficulty paying for college? Pers Soc Psychol Bul 1991;17:606-611.

27 Avila R, Borna S: Discrimination against obese sales personnel. In press. Ball State University, www.bsu.edu/news/article/0,1370,31579--,00.html.

28 Hebl MR, Dovidio JF: Promoting the 'social' in the examination of social stigmas. Pers Soc Psychol Rev 2005;9:156-182.

29 Pingitore R, Dugoni BL, Tindale RS, Spring, B: Bias against overweight job applicants in a simulated employment interview. J Appl Soc Psychol 1994;79:909-917.

30 Hebl MR, Mannix LM: The weight of obesity in evaluating others: a mere proximity effect. Pers Soc Psychol Bull 2003;29:28-38.
1 Shapiro JR, King EB, Quiñones MA: Expectations of obese trainees: how stigmatized trainee characteristics influence training effectiveness. J Appl Soc Psychol 2007;92:239-249.

32 Puhl RM, Schwartz MB, Brownell KD: Impact of perceived consensus on stereotypes about obese people: a new approach for reducing bias. Health Psychol 2005;24:517-525.

33 Crocker J, Cornwell B, Major B: The stigma of overweight: affective consequences of attributional ambiguity. J Pers Soc Psychol 1993;64:60-70.

34 Miller CT, Rothblum ED, Felicio D, Brand P: Compensating for stigma: obese and nonobese women's reactions to being visible. Pers Soc Psychol Bull 1995;21:1093-1106.

35 King EB, Shapiro JR, Hebl MR, Singletary SL, Turner S: The stigma of obesity in customer service: a mechanism for remediation and bottom-line consequences of interpersonal discrimination. J Appl Psychol 2006;91:579-593.

36 Morrison TG, Roddy S, Ryan TA: Methods for measuring attitudes about obese people; in Allison DB, Baskin ML (eds): Handbook of Assessment Methods for Obesity and Eating Behaviors. Thousand Oaks, CA, Sage Publications, 2009, pp. 79-114.

37 Schwartz MB, Chambliss HO, Brownell KD, Blair $\mathrm{SN}$, Billington C: Weight bias among health professionals specializing in obesity. Obes Res 2003;11: 1033-1039.

38 Brochu PM, Morrison MA: Implicit and explicit prejudice toward overweight and average-weight men and women: testing their correspondence and relation to behavioral intentions. J Soc Psychol 2007;147:681-706.
39 Dovidio JF, Kawakami K, Johnson C, Johnson B, Howard A: On the nature of prejudice: automatic and controlled processes. J of Exp Soc Psychol 1997;33:510-540.

40 Krendl AC, Macrae CN, Kelley WM, Fugelsang JA, Heatherton TF: The good, the bad, and the ugly: an fMRI investigation of the functional anatomic correlates of stigma. Soc Neurosci 2006;1:5-15.

41 Pai MP, Paloucek FP: The origin of the 'Ideal' body weight equations. Ann Pharmacol 2000;34 1066-1069.

42 Prentice AM, Jebb SA: Beyond body mass index. Obes Rev 2001:2:141-147.

43 Smith CA, Schmoll K, Konik J, Oberlander S Carrying weight for the world: influence of weight descriptors on judgments of large-sized women. J Appl Soc Psychol 2007;37:989-1006.

44 Finkelstein LM, Demuth RLF, Sweeney DL: Bias against overweight job applicants: further explorations of when and why. Hum Resour Manage 2007;46:203-222.

45 Roehling MV, Roehling PV, Pichler S: The relationship between body weight and perceived weight-related employment discrimination: the role of sex and race. J Vocat Behav 2007;71:300-318.

46 Shelton N, Dovidio, JF, Hebl M, Richeson J: Prejudice and intergroup interaction; in Demoulin $\mathrm{S}$ Leyens JP, Dovidio JF (eds): Intergroup Misunderstandings: Impact of Divergent Social Realities. New York, Psychology Press, 2008.

47 Richeson J, Dovidio JF, Shelton N, Hebl M: Implications for ingroup-outgroup membership for interpersonal perceptions: faces and emotions; in Hess U, Kirouac G (eds): Group Dynamics and Emotional Expression, 3rd ed. New York, Guilford Press, 2007, pp. 7-32. 